\title{
Charge Density Analysis and Transport Properties of TTF Based Molecular Nanowires: A DFT Approach
}

\author{
Karuppannan Selvaraju, ${ }^{1,2}$ and Poomani Kumaradhas ${ }^{1}$ \\ ${ }^{1}$ Department of Physics, Periyar University, Salem 636011, India \\ ${ }^{2}$ Department of Physics, Kandaswami Kandar's College, Velur 638182, India \\ Correspondence should be addressed to Poomani Kumaradhas; kumaradhas@yahoo.com
}

Received 22 July 2014; Accepted 13 November 2014

Academic Editor: Emilio Muñoz-Sandoval

Copyright (c) $2015 \mathrm{~K}$. Selvaraju and P. Kumaradhas. This is an open access article distributed under the Creative Commons Attribution License, which permits unrestricted use, distribution, and reproduction in any medium, provided the original work is properly cited.

\begin{abstract}
The present study has been performed to understand the charge density distribution and the electrical characteristics of Au and thiol substituted tetrathiafulvalene (TTF) based molecular nanowire. A quantum chemical calculation has been carried out using DFT method (B3LYP) with the LANL2DZ basis set under various applied electric fields (EFs). The bond topological analysis characterizes the terminal $\mathrm{Au}-\mathrm{S}$ and $\mathrm{S}-\mathrm{C}$ bonds as well as all the bonds of central TTF unit of the molecule. The variation of electron density and Laplacian of electron density at the bond critical point of bonds for zero and different applied fields reveal the electron density distribution of the molecule. The molecular conformation, the variation of atomic charges and energy density distribution of the molecule have been analyzed for the various levels of applied EFs. The HOMO-LUMO gap calculated from quantum chemical calculations has been compared with the value calculated from the density of states. The variation of dipole moment due to the polarization effect and the $I-V$ characteristics of the molecule for the various applied EFs have been well discussed.
\end{abstract}

\section{Introduction}

Molecular scale electronics is known as single molecule electronics and it is a branch of nanotechnology. Mostly, it deals with single molecules or nanoscale collections of single molecules, which can be used as basic electronic components for fabricating molecular level devices. The ultimate aim of molecular electronics is miniaturization of electrical circuits. This can be done by using single molecules, which constitute the smallest stable structures [1-4]. In the recent years, large efforts have been made to understand structural and electron transport properties of such molecules [5-7], which are currently being used in electronics as interconnects, diodes, switches, rectifiers, transistors, nonlinear components, dielectrics, and memories [8-11]. Generally, higher conductivities originate from highly conjugated molecular systems [12-14]. Tetrathiafulvalene (TTF) is a heterocyclic organosulfur compound which has distinctive electrical properties; notably, it exhibits a high anisotropic electrical conductivity. In addition, TTF is a well-known $\pi$-electron donor in the field of organic metals [15-17]. Studies on this compound contribute to the development of molecular electronics. Bulk TTF itself has unremarkable electrical properties. Distinctive properties are, however, associated with salts of its oxidized derivatives [15-19]. The high electrical conductivity of TTF salts can be attributed to its high symmetry, which promotes charge delocalization, thereby minimizing columbic repulsions. So far, several scientific reports have discussed the TTF and its derivatives [20-22]. However, this is the first report exploring the charge density distribution, bond topological, electrostatic, and transport properties of $\mathrm{Au}$ and thiol substituted TTF based molecule (Figure 1) under zero bias, and various levels of external applied EFs. Numerous theoretical ideas have been used to understand the conductivity of molecular wires (onedimensional systems). The charge density distribution and electrostatic properties along with the structural information give an accurate outline to design highly conducting new molecules. The wave function obtained from high level $a b$ initio/DFT calculations coupled with Bader's Quantum 


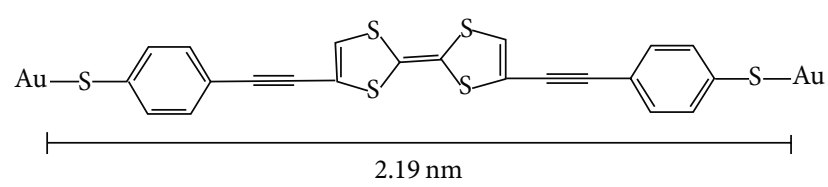

FIgURE 1: $\mathrm{Au}$ and $\mathrm{S}$ substituted tetrathiafulvalene (TTF) based molecule.

theory of atoms in molecules (AIM) $[23,24]$ makes it possible to determine the above properties for zero bias and various applied EFs.

\section{Computational Details}

Understanding the effect of applied electric field on the structural and electronic properties of the Au and thiol substituted TTF based molecule has been analyzed by optimizing it for the zero and applied fields for both directions [five biasing steps $\pm 0.04, \pm 0.08, \pm 0.12, \pm 0.16$ and $\left.\pm 0.20 \mathrm{~V}^{-1}\right]$. All calculations in this study have been carried out using density functional theory (DFT) [23-27]. Gaussian03 program [28] is used throughout all computations. Here, we have used LANL2DZ basis set [29] for whole DFT calculation with B3LYP hybrid function to obtain effective core potential and the detailed description of the effect of heavy metal atoms in the molecule [29-31]. All geometric optimizations have been performed via Berny algorithm in redundant internal coordinates. The self-consistency of noninteractive wave function has been performed with a requested convergence on the density matrix of $10^{-8}$ and $10^{-6}$ for the RMS and maximum density matrix error between the iterations [32]. The wave function obtained from each calculation has been analyzed with the theory of "atoms in molecules" (AIM) and the atomic property has been calculated using AIMPAC program [33].

By using EXT94b routine incorporated to the AIMPAC software, the electron density $\rho_{\mathrm{bcp}}(r)$, Laplacian of electron density $\nabla^{2} \rho_{\text {bcp }}(r)$, and bond ellipticity $\varepsilon$ have been calculated for various applied fields. The programs wfn2plots and DENPROP have been used to plot the deformation and the Laplacian of electron density maps. The electrostatic potential of the molecule has been plotted with Gview [34] to visualize the isosurface of positive and negative ESP regions of the molecule. The density of states (DOS) at various EFs has been determined by using GuassSum program [35]. For the various applied electric fields $(E)$, the bias voltage $(V)$ across the molecule of length $L$ has been calculated from the expression $V=E L$. Further, using Ohm's law $(I=V / R)$, the current $(I)$ flows through the wire has been calculated for each biasing step.

\section{Results and Discussion}

3.1. Structural Aspects. The geometric parameters, especially bond lengths, are important parameters for adjusting the electrical properties of molecular wires. Also, the average difference between the adjacent single and double bonds known as the bond length alternation along the backbone of a conjugated system plays a vital role for tuning the transport properties. Therefore, a detailed study of bond length variation under the EF interaction is instructive for understanding the relationship between molecular structure and property. The optimized geometry of Au and S substituted TTF based molecular wire for the zero bias and the maximum applied EF (0.20) is illustrated in Table 6. (Optimized geometries for various EFs are given in supplementary Figure S1 (see Figure S1 in Supplementary Material available online at http://dx.doi.org/10.1155/2015/806181)). This molecular wire has two aromatic rings with central TTF group and the Au atoms attached at both ends of the molecule through thiol atoms. The thiol atom forms an excellent link [36] between the conjugated TTF molecule and the Au atom.

For the zero field, the $\mathrm{C}-\mathrm{C}$ bond distances of two aromatic rings and the $\mathrm{C} \equiv \mathrm{C}$ bonds that link the rings in the molecule are $\sim 1.41$ and $\sim 1.22 \AA$, respectively. When the field is applied, these distances are slightly modified; however, the EF dependence of bond length evolution is not identical for all the bonds. That is, the maximum observed variation in $\mathrm{C}-\mathrm{C}$ bonds is 0.009 whereas $\mathrm{C} \equiv \mathrm{C}$ bonds are $0.003 \AA$. Also, in most cases it is found that the $\mathrm{C}-\mathrm{C}$ single bonds become shorter and the double and triple bonds become longer resulting in higher conjugation, which is pertinent to reported results $[37,38]$. The zero field distance of S-C bonds in the TTF unit is $\sim 1.83 \AA$; as the field increases, the maximum variation observed is $0.013 \AA$. Similarly, the applied $\mathrm{EF}$ alters the bond distances of terminal S-C bonds, which are found to be unequal on both ends. In the left-end (Lend), the distance increases from 1.838 to $1.842 \AA$, while in the right-end (R-end), the distance decreases from 1.837 to $1.828 \AA$; notably, the variation in the R-end is slightly greater than the L-end and the value is $\sim 0.009 \AA$. Hence, it is found that the $\mathrm{S}-\mathrm{C}$ bond distances increase in the high potential side while they decrease in low potential side. As the field increases, the distance of $\mathrm{Au}-\mathrm{S}$ bond in the $\mathrm{L}$-end decreases from 2.401 to $2.388 \AA$, while in the R-end, the distance increases from 2.401 to $2.463 \AA$; however, the variations in both ends are unequal. And, for the maximum applied field $\left(0.20 \mathrm{VA}^{-1}\right)$ the variations at $\mathrm{L}$ - and $\mathrm{R}$-ends are 0.013 and $0.038 \AA$, respectively. This large difference attributes, the applied field lengthening the $\mathrm{Au}-\mathrm{S}$ bond through by shrinking the $\mathrm{S}-\mathrm{C}$ bond distance in the wire (Table 1) and these bond distances [ $\mathrm{S}-\mathrm{C}$ and $\mathrm{Au}-\mathrm{S}$ bonds] are very close to the previously reported values [36, 38-41]. Even though almost all bond distances vary by the application of external field, specifically, the $\mathrm{S}-\mathrm{C}$ and $\mathrm{Au}-\mathrm{S}$ bonds have uniform and systematic variation. Hence, we plot the variation of $\mathrm{S}-\mathrm{C}$ and $\mathrm{Au}-\mathrm{S}$ bond lengths for different applied EFs with reference to zero fields (Figure 2). The selected values of bond lengths are presented in Table 1 and the complete values of bond lengths are listed in supplementary Table S1.

3.2. Charge Density Distribution. The selected bond electron density values of TTF based molecular wire for zero and various levels of applied EF are listed in Table 2. Table 7 


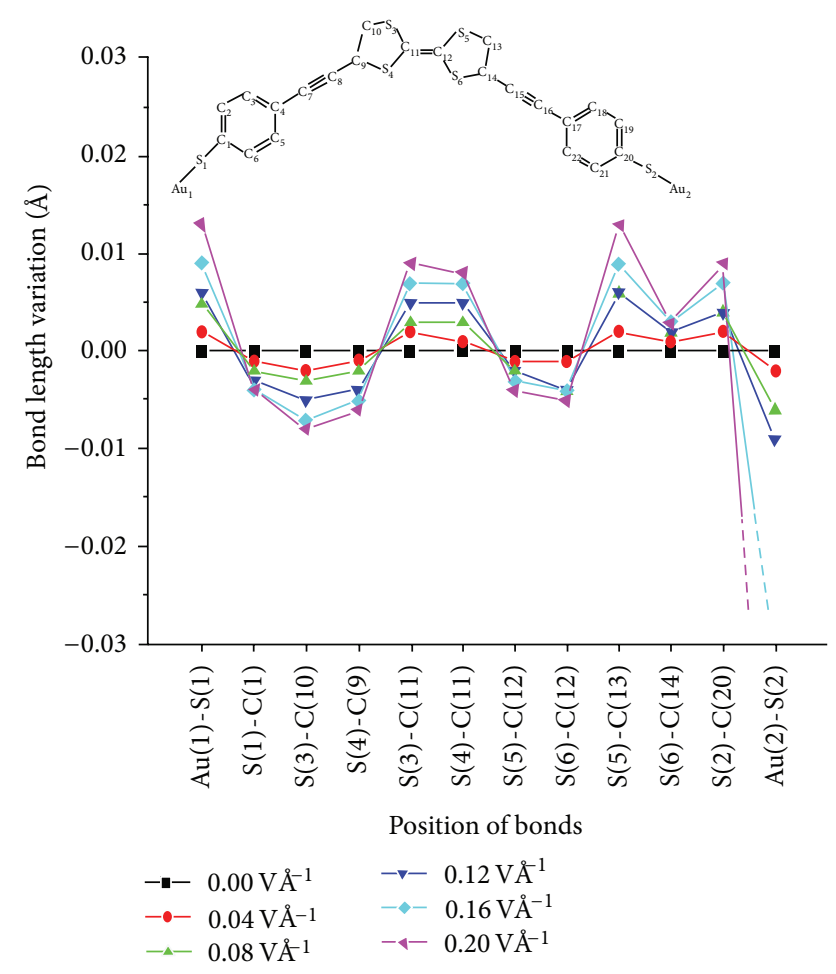

Figure 2: Bond length variations of Au and S substituted TTF based molecule for various applied EFs with reference to zero field.

TABLE 1: Bond lengths ( $\AA$ ) of terminal bonds of $A u$ and $S$ substituted TTF based molecule for zero and various applied EFs $\left(V \AA^{-1}\right)$.

\begin{tabular}{lcccccc}
\hline \multirow{2}{*}{ Bonds } & \multicolumn{7}{c}{ Applied electric field } \\
& 0.00 & 0.04 & 0.08 & 0.12 & 0.16 & 0.20 \\
\hline $\mathrm{S}(1)-\mathrm{C}(1)$ & 1.838 & 1.839 & 1.84 & 1.841 & 1.842 & 1.842 \\
$\mathrm{~S}(2)-\mathrm{C}(20)$ & 1.837 & 1.835 & 1.833 & 1.833 & 1.830 & 1.828 \\
$\mathrm{Au}(1)-\mathrm{S}(1)$ & 2.401 & 2.399 & 2.396 & 2.395 & 2.392 & 2.388 \\
$\mathrm{Au}(2)-\mathrm{S}(2)$ & 2.401 & 2.403 & 2.407 & 2.410 & 2.433 & 2.463 \\
\hline
\end{tabular}

TABLE 2: Electron density $\rho_{\mathrm{bcp}}(r)\left(\mathrm{e} \AA^{-3}\right)$ values of terminal bonds of $\mathrm{Au}$ and $\mathrm{S}$ substituted TTF based molecule for zero and various applied EFs $\left(V \AA^{-1}\right)$.

\begin{tabular}{lcccccc}
\hline \multirow{2}{*}{ Bonds } & \multicolumn{7}{c}{ Applied electric field } \\
& 0.00 & 0.04 & 0.08 & 0.12 & 0.16 & 0.20 \\
\hline $\mathrm{S}(1)-\mathrm{C}(1)$ & 1.017 & 1.015 & 1.013 & 1.010 & 1.010 & 1.009 \\
$\mathrm{~S}(2)-\mathrm{C}(20)$ & 1.018 & 1.021 & 1.024 & 1.025 & 1.027 & 1.028 \\
$\mathrm{Au}(1)-\mathrm{S}(1)$ & 0.520 & 0.522 & 0.525 & 0.528 & 0.532 & 0.538 \\
$\mathrm{Au}(2)-\mathrm{S}(2)$ & 0.520 & 0.517 & 0.514 & 0.511 & 0.489 & 0.465 \\
\hline
\end{tabular}

displays the deformation density maps of TTF based molecular wire, showing the charge accumulation for zero bias and maximum applied field and the differences. The relation between the topology of electron density and the chemical concepts of molecules can be accurately quantified [23] by using Quantum theory of atoms in molecules (QTAIM). The critical point search in the molecule found a $(3,-1)$ type of bond critical point (bcp) for all bonds, which implies
TABLE 3: Laplacian of electron density $\nabla^{2} \rho_{\mathrm{bcp}}(r)\left(\mathrm{e} \AA^{-5}\right)$ values of terminal bonds of $\mathrm{Au}$ and $\mathrm{S}$ substituted TTF based molecule for zero and various applied EFs $\left(\mathrm{V} \AA^{-1}\right)$.

\begin{tabular}{lcccccc}
\hline \multirow{2}{*}{ Bonds } & \multicolumn{7}{c}{ Applied electric field } \\
& 0.00 & 0.04 & 0.08 & 0.12 & 0.16 & 0.20 \\
\hline Terminal bonds \\
$\mathrm{S}(1)-\mathrm{C}(1)$ & -4.207 & -4.186 & -4.167 & -4.142 & -4.123 & -4.101 \\
$\mathrm{~S}(2)-\mathrm{C}(20)$ & -4.217 & -4.253 & -4.297 & -4.301 & -4.345 & -4.387 \\
$\mathrm{Au}(1)-\mathrm{S}(1)$ & 2.950 & 2.971 & 3.014 & 3.024 & 3.218 & 3.409 \\
$\mathrm{Au}(2)-\mathrm{S}(2)$ & 2.954 & 2.947 & 2.925 & 2.925 & 2.925 & 2.925 \\
\hline
\end{tabular}

that the chemical bonds $[42,43]$ exist in the molecule. We have also found that the interaction between $\mathrm{Au}$ and $\mathrm{S}$ atoms in $\mathrm{Au}-\mathrm{S}$ bond of TTF based molecular wire is not a covalent interaction; hence, the $\mathrm{Au}-\mathrm{S}$ bond is a very weak coordination bond. Also, the positive Laplacian of electron density of Au-S bond (Table 3) shows the existence of closed shell interaction between the S and Au atoms; this confirms the noncovalent interaction of $\mathrm{Au}-\mathrm{S}$ bonds which is applicable to the reported results [44-46].

The zero field electron density $\left[\rho_{\mathrm{bcp}}(r)\right]$ at the bcp of all aromatic C-C bonds ranges from $\sim 1.884$ to $\sim 1.949 \mathrm{e}^{-3}$, whereas for the applied field these values are slightly varied, and the maximum variation is $0.037 \mathrm{e}^{-3}$. Similarly, the zero field electron density $\rho_{\mathrm{bcp}}(r)$ of the $\mathrm{C}-\mathrm{C}$ bonds connecting the two thiophene rings in the TTF unit is $\sim 2.092 \mathrm{e}^{-3}$; as the field increases, this value decreases and the observed maximum electron density variation of the bond is $0.019 \mathrm{e}^{-3}$. The zero field density of $\mathrm{C}-\mathrm{H}$ bond is $\sim 1.8 \mathrm{e} \AA^{-3}$; this value is not much altered in the presence of electric field. The $\mathrm{S}-\mathrm{C}$ bond electron density of thiophene rings for the zero field ranges from $\sim 0.996$ to $1.084 \mathrm{e}^{-3}$ and for the applied field, it decreases; the maximum variation observed is $0.024 \mathrm{e}^{-3}$. The $\mathrm{C} \equiv \mathrm{C}$ bonds exhibit high electron density for the zero bias $\left(2.477 \mathrm{e}^{-3}\right)$, and for the applied field, the variation is found to be very small. On comparing the $\rho_{\mathrm{bcp}}(r)$ values of $\mathrm{C}-\mathrm{C}, \mathrm{C}-\mathrm{H}$, and $\mathrm{S}-\mathrm{C}$ bonds, the density of $\mathrm{S}-\mathrm{C}$ bond is notably small. This indicates that the charges of these bonds are moving away from the internuclear axis, which confirms its dominant $\pi$-bond nature [40]. Also, this can be well understood from the Laplacian of electron density of the molecule compared with the Cremer and Kraka's work $[42,43]$. The Au-S bond density at zero field is $0.52 \mathrm{e}^{-3}$, whereas for the applied field the density increases to $0.538 \mathrm{e}^{-3}$ in the L-end, but in R-end it decreases to $0.476 \mathrm{e} \AA^{-3}$; Although the electron density of $\mathrm{Au}-\mathrm{S}$ bond is very small, the observed variation $\left(0.055 \mathrm{e}^{-3}\right)$ is greater than all other bonds. The effect of electric field in the molecule is not much altered the electron densities of the bond in the molecule. Relatively, the variations are small for the applied field and are found to be very systematic (Supplementary Table S2). The increase or decrease of applied field in the molecule did not make any significant change in the bond charge accumulation of the molecule. 
The Laplacian of the electron density $\left[\nabla^{2} \rho_{\mathrm{bcp}}(r)\right]$ allows to understand the charge concentration or depletion at the bcp [42]. It plays significant role in the study of the charge density $[47,48]$. In this work, the Laplacian values for all bonds in the molecule have been calculated to realize whether the charges at the bcp of the bonds are concentrated or depleted when the molecules is exposed to external EFs. The selected Laplacian of electron density for the various applied EFs is shown in Table 3. Table 8 shows the Laplacian of electron density maps for zero and the maximum applied field $\left(0.20 \mathrm{~V}^{-1}\right)$. For the zero field, the predicted Laplacian of electron density for the aromatic $\mathrm{C}-\mathrm{C}$ bonds ranges from -17.3 to $-18.5 \mathrm{e}^{-5}$, whereas for the applied field these values become little less negative, indicating the charges of these bonds are slightly depleted (Supplementary Figure S2). Similar trend is found in the $\mathrm{C}-\mathrm{C}$ bonds, which are connecting the rings in the molecule, in which Laplacian for zero field ranges from -16.3 to $-19.9 \mathrm{e} \AA^{-5}$, whereas for the applied field, the maximum variation observed is $0.314 \mathrm{e}^{-5}$. The zero field Laplacian of electron density for the $\mathrm{C}-\mathrm{H}$ bonds is $\sim-20.5 \mathrm{e}^{-5}$, the high negative value of Laplacian, which indicates the charge concentration, and the applied field slightly alters this charge concentration. The Laplacian for the terminal S-C bonds of L-end and R-end is -4.207 and $-4.217 \mathrm{e}^{-5}$, respectively. As the field increases, the Laplacian value in the L-end decreases to $-4.101 \mathrm{e}^{-5}$ and in the R-end, it increases to $-4.387 \mathrm{e} \AA^{-5}$. For the zero field, the Laplacian of $\mathrm{Au}-\mathrm{S}$ bond is $\sim 2.95 \mathrm{e}^{-5}$; when the field increases, this value slightly increases to $3.409 \mathrm{e}^{-5}$ at the L-end, but at the R-end it decreases to $2.925 \mathrm{e}^{-5}$. Overall, the Laplacian of electron density distribution in the Au substituted molecular wire $(\mathrm{Au}-\mathrm{S}-$ molecule-S$-\mathrm{Au}$ system) reveals that the applied field depletes the charges at the bcps of $\mathrm{C}-\mathrm{C}$ bonds, whereas this effect is found little more in the terminal bonds (Supplementary Figure S3); specifically it is high at the R-end. The complete values of Laplacian of electron density for the various applied EFs are shown in supplementary Table S3.

3.3. Energy Density. Bond energy density is the measure of bond strength in a chemical bond. The chemical bond is a fundamental concept, which provides an important basis for rationalizing the structural properties, stability, and reactivity for a host of materials. In addition to the bond critical point properties, the calculated energy density distributions provide important information about the local energy density properties for the bonded interactions [49]. Further, the energy density distribution of TTF based molecule is directly related to Laplacian of electron density [40, 42, 43]. When the Laplacian of electron density is positive, the kinetic energy density is dominant, which leads to the depletion of bond charge; if it is negative, the potential energy density dominates, and the accumulation of charge is expected to happen $[25,42,43]$. Also, the kinetic energy density analysis of TTF identifies patterns within its electronic structure, which are linked to familiar concepts of chemical bonding [50]. The total energy density in the bonding region $H(\mathbf{r})$ is expressed as $H(\mathbf{r})=G(\mathbf{r})+V(\mathbf{r})$, where $V(\mathbf{r})$ is the potential energy density and $G(\mathbf{r})$ is the local kinetic energy

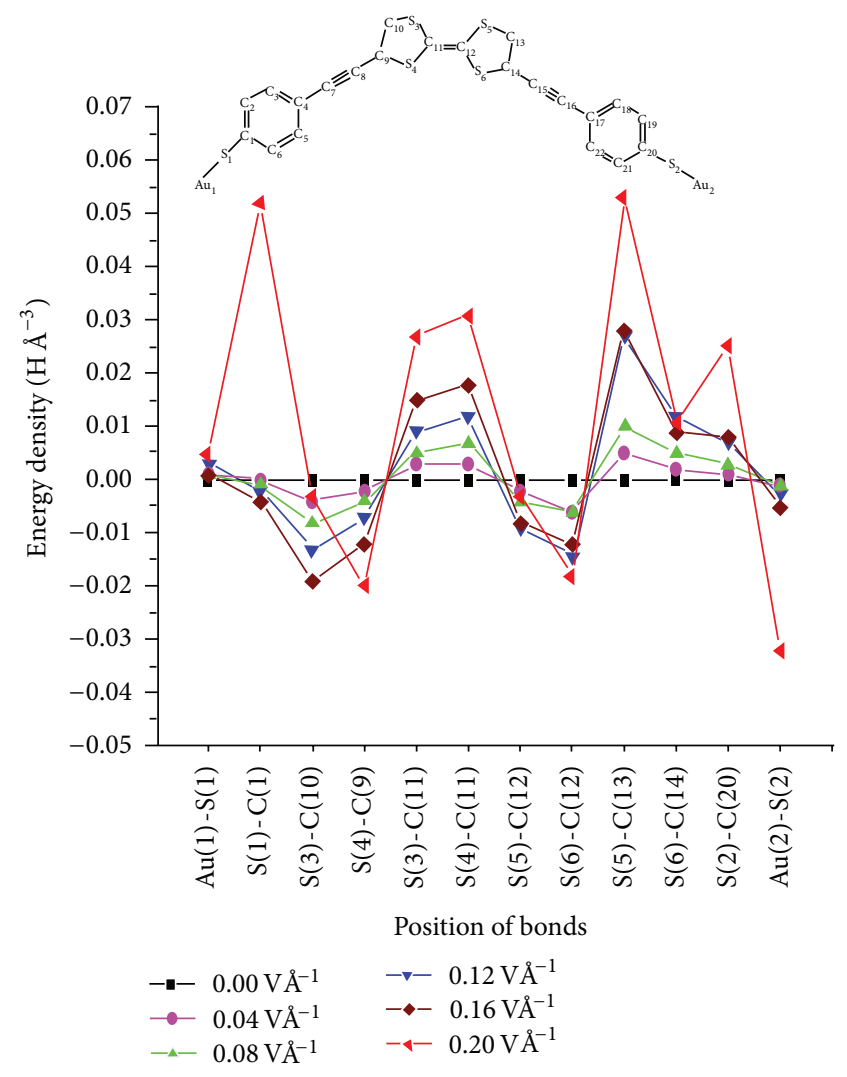

Figure 3: Energy density variations of Au and S substituted TTF based molecule for various applied EFs with reference to zero field.

density [40]. In the case of TTF based molecule, $G(\mathbf{r})$ is positive, $V(\mathbf{r})$ is negative, and the total energy density $H(\mathbf{r})$ is negative, which indicates that $V(\mathbf{r})$ dominates for all cases. The calculated zero field energy density $H(\mathbf{r})$ for the $\mathrm{C}-\mathrm{C}$ bond in the aromatic ring is highly negative, which ranges from -1.813 to $-1.944 \mathrm{H}^{-3}$; when the applied EF increases, these values are slightly decreased within short range $(-1.789$ to $-1.936 \mathrm{HA}^{-3}$ ).

The zero field energy density $H(\mathbf{r})$ for the $\mathrm{C}-\mathrm{C}$ bonds in the TTF unit is $-2.212 \mathrm{H}^{-3}$; when the field increases, this value decreases to $-2.178 \mathrm{H} \AA^{-3}$. The energy density of $\mathrm{C}(11)-\mathrm{C}(12)$ bond connecting the rings varies from -2.268 to $-2.226 \mathrm{H}^{-3}$. Notably, the $\mathrm{C} \equiv \mathrm{C}$ bond in the molecule exhibits the high energy density $\left(-3.217 \mathrm{H}^{-3}\right)$ for the maximum applied field $\left(0.20 \mathrm{~V}^{-1}\right)$. The energy densities $H(\mathbf{r})$ for the $\mathrm{C}-\mathrm{H}$ bonds for different fields range from -1.722 to $-1.767 \mathrm{H}^{-3}$. Notably, the energy density distribution in the terminal heavy atom bonds ( $\mathrm{Au}-\mathrm{S}$ and $\mathrm{S}-\mathrm{C}$ ) is significantly less $\left(-0.156\right.$ and $\left.-0.62 \mathrm{H}^{-3}\right)$ in comparison with the other bonds in the molecule. The small values are due to the nature of bonds. Further, for the applied field, the variation between both types of bonds is found to be opposite. However, the variations of $\mathrm{S}-\mathrm{C}$ and $\mathrm{Au}-\mathrm{S}$ bonds are significant and systematic. Figure 3 shows the energy density variations of the molecule. The calculated values of energy density distribution of the terminal bonds of the molecule for zero and various 
TABLE 4: Bond energy density $\left(H \AA^{-3}\right)$ values of terminal bonds of $\mathrm{Au}$ and $\mathrm{S}$ substituted TTF based molecule for the zero and various applied EFs $\left(V \AA^{-1}\right)$.

\begin{tabular}{lcccccc}
\hline \multirow{2}{*}{ Bonds } & \multicolumn{7}{c}{ Applied electric field } \\
& 0.00 & 0.04 & 0.08 & 0.12 & 0.16 & 0.20 \\
\hline Terminal bonds \\
$\mathrm{S}(1)-\mathrm{C}(1)$ & -0.619 & -0.618 & -0.617 & -0.615 & -0.616 & -0.671 \\
$\mathrm{~S}(2)-\mathrm{C}(20)$ & -0.621 & -0.624 & -0.628 & -0.629 & -6.400 & -0.646 \\
$\mathrm{Au}(1)-\mathrm{S}(1)$ & -0.156 & -0.157 & -0.159 & -0.157 & -0.146 & -0.161 \\
$\mathrm{Au}(2)-\mathrm{S}(2)$ & -0.156 & -0.155 & -0.153 & -0.151 & -0.144 & -0.124 \\
\hline
\end{tabular}

TABle 5: Atomic charges (e) of terminal atoms for the zero and various applied electric fields (first line CHELPG charges, second line MK charges).

\begin{tabular}{ccccccc}
\hline \multirow{2}{*}{ Atom } & \multicolumn{7}{c}{ Applied electric field $\left(\AA^{-1}\right)$} \\
& 0.00 & 0.04 & 0.08 & 0.12 & 0.16 & 0.20 \\
\hline \multirow{2}{*}{$\mathrm{S}(1)$} & -0.333 & -0.332 & -0.332 & -0.331 & -0.331 & -0.331 \\
& -0.302 & -0.303 & -0.302 & -0.307 & -0.307 & -0.293 \\
\hline \multirow{2}{*}{$\mathrm{S}(2)$} & -0.333 & -0.333 & -0.333 & -0.333 & -0.333 & -0.348 \\
& -0.303 & -0.303 & -0.303 & -0.307 & -0.307 & -0.310 \\
\hline \multirow{2}{*}{$\mathrm{Au}(1)$} & 0.175 & 0.177 & 0.177 & 0.179 & 0.181 & 0.183 \\
& 0.156 & 0.157 & 0.157 & 0.159 & 0.161 & 0.165 \\
\hline \multirow{2}{*}{$\mathrm{Au}(2)$} & 0.177 & 0.177 & 0.177 & 0.176 & 0.176 & 0.176 \\
& 0.159 & 0.158 & 0.158 & 0.158 & 0.156 & 0.155 \\
\hline
\end{tabular}

applied EFs are presented in Table 4 and the complete values are given in supplementary Table S4.

3.4. Atomic Charges. To determine the atomic charges, various methods are available; the most frequently used are natural population analysis, Mulliken population analysis, Chelpg scheme, and Merz-kollman (MK) schemes, which express the electrostatic interactions more precisely. The scheme of point charge distribution of molecules plays a major role in understanding the chemical reactivity and electrostatic potential [51-53]. The Chelpg charges are consistent with the electrostatic Poisson equation. Further, a number of studies have shown that MK method provides the best values according to electrostatic criteria [53, 54]. Both Chelpg and MK schemes are grid based methods, in which the atomic charges are fitted to reproduce the molecular electrostatic potential (MEP) at a number of points around the molecule $[54,55]$. Hence, in the present work, we have calculated the point charges by Chelpg and MK schemes.

The Chelpg charges of all C-atoms except those which are linked to $\mathrm{S}$ atoms possess negative charge and vary with the increase of field. The linker S(1)-atom possesses negative Chelpg charge, which decreases from -0.333 to $-0.331 \mathrm{e}$ with increase of field, while the charge of S(2)-atom increases from -0.333 to -0.348 e. As the field increases, the charges of $\mathrm{Au}$ atom at L-end slightly increase from 0.175 to $0.183 \mathrm{e}$, but the same at the R-end almost remains the same $(0.177,0.176 \mathrm{e})$. For the zero field, the MK charge for all C-atoms is found almost negative, and the $\mathrm{H}$-atoms are positive; when the field increases, the charge of the atoms also found increases.
For the applied field, the MK charges of S-atom at the Lend decrease gradually from -0.302 to $-0.293 \mathrm{e}$, while at the $\mathrm{R}$-end this effect is opposite and increases from -0.303 to $-0.310 \mathrm{e}$. As the field increases, the charge of $\mathrm{Au}(1)$ atom increases from 0.156 to $0.165 \mathrm{e}$, but the same for $\mathrm{Au}(2)$ slightly decreases from 0.159 to 0.155 e (Table 5). The differences of charge distribution for zero and various applied EFs are presented in supplementary Table S5.

3.5. Molecular Orbital Analysis. Generally, for any molecular level device, the charge transport characteristics are mainly controlled by the nature of the molecular orbitals. The spatial distribution and the energy level of a molecular orbital (MO) determine its contribution to the conductivity $[56,57]$. The charge transfer through a particular MO gradually decreases as we go away from the Fermi level of the electrode. Further, the MOs, which are fully delocalized, contribute more to conduction channel [58-61]. The frontier molecular orbitals are the highest occupied molecular orbital (HOMO) and lowest unoccupied molecular orbital (LUMO) and the difference between them is known as HOMO-LUMO gap (HLG). Recently, several theoretical studies report the influence of HOMO-LUMO gaps and the spatial distributions of molecular orbitals on the electronic transport through the molecular device $[62,63]$. Further, the charge transport properties of the molecule [64] are determined by the difference of energy between HOMO and LUMO. Hence, it is essential to examine the variations of HLG and molecular orbital energy levels [64-66] for the various applied EFs. Table 9 shows the spatial redistribution of molecular orbital of TTF for the zero bias and the maximum applied EF $\left(0.20 \mathrm{~V}^{-1}\right)$. The applied EFs partially localize the frontier orbitals (HOMO-2, HOMO-1, HOMO, LUMO, LUMO+1, and LUMO+2) of the molecules, which are opposite to each other; this can be well understood from Table 9.

For the applied fields $\left(0-0.20 \mathrm{~V} \AA^{-1}\right)$, the HLG decreases from 1.486 to $0.218 \mathrm{eV}$. This variation is also confirmed from the spectrum of density of states (DOS). Figures 4(a) and 4(b) show the DOS of Au substituted molecule, in which the HOMO (green lines) and the LUMO (blue lines) and the HLG are shown. Notably, the presence of gold atoms in the molecule broadens the DOS peaks. Seemingly, the significant decrease of HLG may facilitate large electron conduction $[67,68]$ through the molecule; hence, the $\mathrm{Au}$ substituted TTF based molecule can perform as an efficient molecular nanowire. Figure 5 represents the energy levels of the molecule for various applied EFs.

3.6. Electrostatic Potential. Molecular electrostatic surface potential (ESP) is another piece of information which is required to understand the electronic properties of molecules $[69,70]$. Areas of the molecule with specific properties such as electron donation or electron-withdrawing capabilities and their stabilities can be easily evaluated from an ESP map [71]; this information is extremely useful in understanding molecular interactions, which helps to design molecular electronic devices $[72,73]$. The isosurface representation of ESP of Au and S substituted TTF based molecule for 


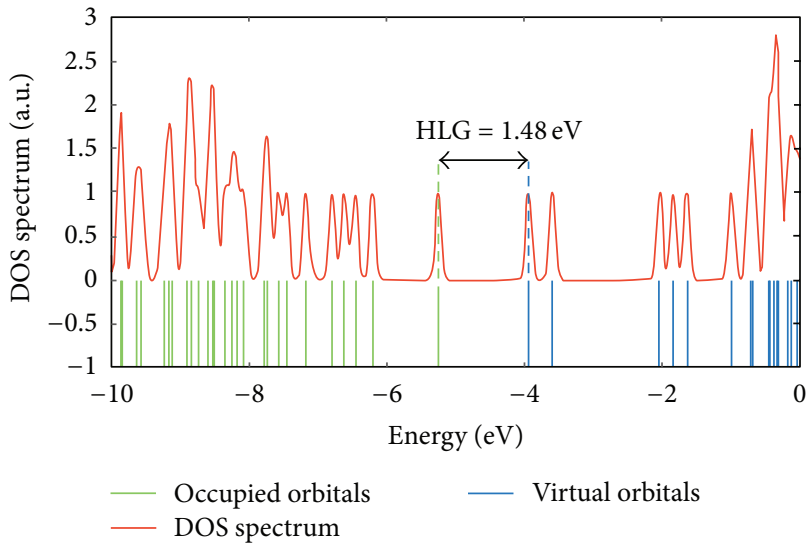

(a)

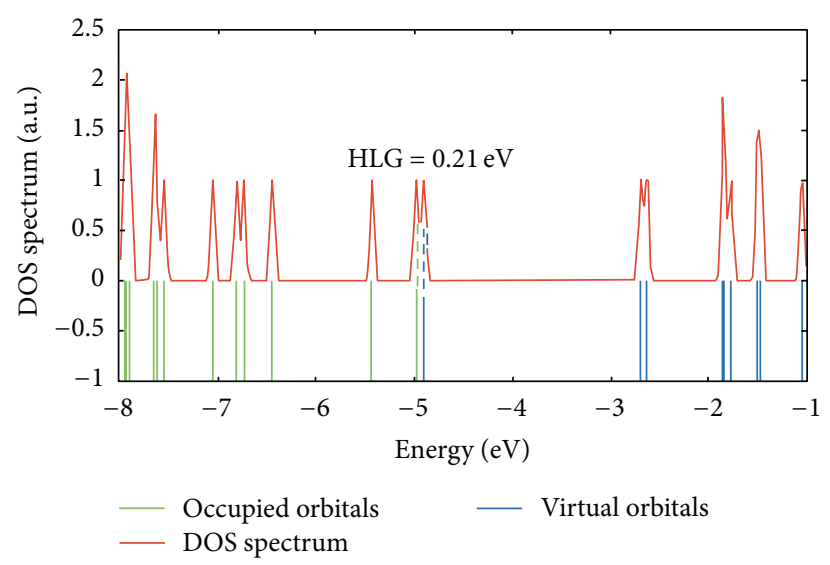

(b)

FIGURE 4: DOS of Au and S substituted TTF based molecule for (a) zero and (b) maximum applied EF $\left(0.20 \mathrm{~V}^{-1}\right)$.



Figure 5: Energy level diagram of Au and S substituted TTF based molecule for the zero and various applied EFs.

the various applied EFs is shown in Table 10. The Au-S bond regions exhibit high negative ESP, which are the negative charged regions (red) of the molecule, and it explicitly reflects the opposing contributions from the nuclei and the electrons.

For the zero bias, the negative ESP is concentrated around the S-atoms, which are present at either ends of the molecule and also $S$ atoms in the TTF unit. The rest of the molecule carries positive ESP. For the increase of positive field from $0-0.20 \mathrm{~V}^{-1}$, the negative ESP at the L-end of the molecule gradually decreases for each biasing step and it disappears, while the same at the R-end gradually increases and finally spreads around the right edge of the molecule (Table 10); this shows that when the field increases the charges seem to drift from left to right. Similarly, the negative ESP regions are moved from R- to L-end of the molecule when the field is reversed. The ESP map clearly shows the effect of substitution and the applied EFs in molecule.

3.7. Molecular Dipole Moment. When the molecule is subjected to an external EF, the delocalization of $\pi$-electron of



FIgURE 6: Molecular dipole moment of Au and S substituted TTF based molecule for the zero and various applied EFs.

the conjugated organic molecules leads to redistribution of charges of the molecular chain, and consequently, the dipole moment of the molecule changes $[38,74,75]$. Hence, we can roughly estimate the ability of electron transport by simply comparing the dipole moments of the molecule for various applied EFs. The variations of molecular dipole moment for the various applied EFs were analyzed by Kirtman et al. [76] and found a linear character. However, this linearity no longer exists beyond certain applied field and it is unimportant since no molecular electronic device works under such high voltages [77]. Here, we have calculated the dipole moment of the molecule for zero bias as well as various applied EFs. The resultant molecular dipole moment $(\mu)$ for zero bias is $1.31 \mathrm{D}$, which increases almost linearly with the increase of field. The molecule becomes highly polarized for the higher field $\left(0.20 \mathrm{~V} \AA^{-1}\right)$ and the dipole moment becomes $35.9 \mathrm{D}$. Figure 6 shows the variation of $x, y$, and $z$ components of dipole moment $\left(\mu_{x}, \mu_{y}\right.$, and $\left.\mu_{z}\right)$ and the resultant molecular 
TABLE 6: Optimized geometry of Au and S substituted TTF based molecule for the zero and maximum applied EF $0.20 \mathrm{~V} \AA^{-1}$.

$\mathrm{EF}\left(\mathrm{V}^{-1}\right)$

TABLe 7: Deformation density maps of Au and S substituted TTF based molecule for the zero and maximum applied EF $0.20 \mathrm{~V} \AA^{-1}$. Solid lines represent positive contours, dotted lines are negative contours, and dashed lines are zero contours. The contours are drawn at $0.05 \mathrm{e}^{-3}$ intervals.

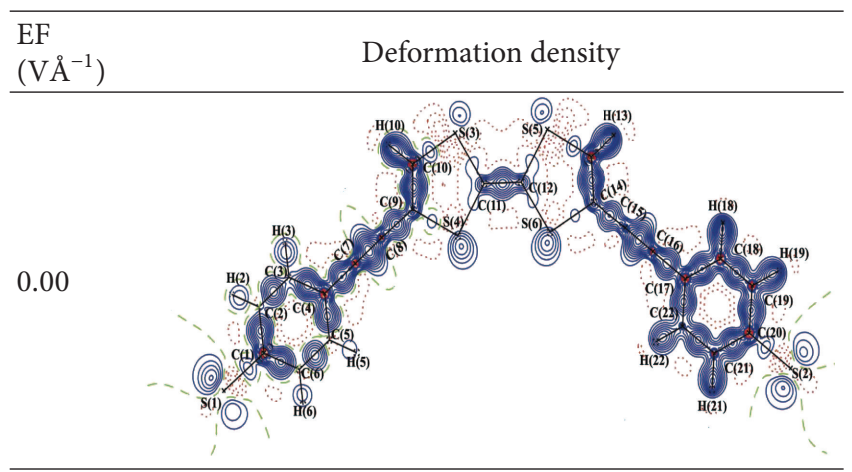

0.20

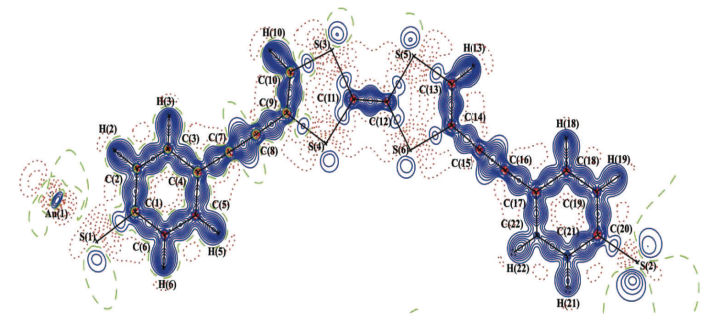

dipole moment $(\mu)$ for various applied EFs, the large variation of $x$-component [68], may be due to the application of field along $x$-direction.

3.8. I-V Characteristic Curve. As a preliminary approach for calculating the $I-V$ characteristic of single molecules, a purely $a b$ initio approach was developed [78]. This method uses molecular calculations to estimate the $I-V$ through a molecule. Thus formulating a new prescription, one can obtain current-voltage characteristics via the use of precise quantum chemistry techniques. Here, we have evaluated the $I-V$ characteristics of the TTF based molecular wire using
TABLE 8: Laplacian of electron density maps of $\mathrm{Au}$ and $\mathrm{S}$ substituted TTF based molecule for the zero and maximum applied $\mathrm{EF} 0.20 \mathrm{~V} \AA^{-1}$. The contours are drawn in logarithmic scale, $3.0 \times 2^{N}$ e $\AA^{-5}$, where $N=2,4$ and $8 \times 10^{n}, n=-2,-1,0,1,2$. Solid lines are positive contours and dotted lines are negative contours.



0.20



the Landauer formula [79]. The tunneling electric current $(I)$ has been calculated for various applied electric fields $(E)$ and the bias voltage $(V)$ across the molecule of length $L$. The linear conductance $(G)$ and the resistance $(R)$ of the electrode-molecule-electrode junctions can be expressed as

$$
R=G^{-1}=\left(\frac{h}{2 e^{2}}\right)\left(\frac{1}{T_{l} T_{r} T_{m}}\right)=\frac{12.91 \mathrm{~K} \Omega}{T_{l} T_{r} T_{m}},
$$

where $T_{l}$ and $T_{r}$ are the charge transport efficiency across the left and right contacts, $T_{m}$ is the electron transmission 
TABLE 9: Isosurface representation of molecular orbitals of Au and S substituted TTF based molecule for the zero and maximum applied electric field $\left(0.20 \mathrm{~V}^{-1}\right)$, which are drawn at 0.05 au surface values.

EF (VA $\left.{ }^{-1}\right)$



FIgURE 7: $I-V$ Characteristics of Au and S substituted TTF based molecule for various applied EFs.

through the molecule itself, and $\left(h / 2 e^{2}\right)=12.91 \mathrm{~K} \Omega$ is the quantum of resistance [80-83]. The left and right contacts electron transmission $T_{l}$ and $T_{r}$ can be neglected since there is no charge injection barrier in the molecule. $T_{m}$ can be approximated by the expression

$$
T_{m}=\exp (-\beta L)
$$

where $L$ is the potential barrier width which is equivalent to molecular length and $\beta$ is the tunneling decay parameter which can be determined by

$$
\beta=\left(\frac{1}{\hbar}\right)\left[2 m^{*} \alpha \phi\right]^{1 / 2}
$$

where, $m^{*}$ is the effective mass of electron $\left(m^{*}=0.16 m_{0}\right)$, $m_{0}$ is the free electron mass, $\alpha$ is the symmetry parameter of potential profile which is equal to unity for symmetric molecule [80-83], and $\phi$ is the potential barrier height for tunneling through the HOMO or the LUMO level. For a particular external applied EF, $\phi$ is half of the HLG of the molecular wire. Hence, the resistance of the molecular wire has been calculated using expression (1). Further, the bias voltage $(V)$ has been calculated from the expression $V=E L$. Using these parameters, the $I-V$ characteristics of the TTF based molecule have been studied. Figure 7 illustrates the $I-V$ characteristics of TTF based molecule for the various applied EFs, which reveals that as the bias voltage increases, the current increases gradually showing the nonlinear behavior of the molecule. Since the molecule is symmetric, the characteristic curve is also almost symmetric for both directions of the applied EFs.

\section{Conclusion}

The present quantum chemical study on TTF based molecular wire describes the bond topological parameters and the electrical characteristics for zero and various external applied fields. The bond topological analysis shows the variation of electron density $\rho_{\mathrm{bcp}}(r)$ and Laplacian of electron density $\nabla^{2} \rho_{\mathrm{bcp}}(r)$ for zero bias and the various applied fields of the molecule. Systematic and almost uniform redistribution of charge density as well as energy density have been observed for all bonds of the central TTF unit and terminal bonds of the molecule for various applied EFs. When the field increases, the hybridization of molecular levels broadens 
TABLE 10: Molecular electrostatic potential of Au and S substituted TTF based molecule for the zero and various applied EFs. Blue: positive potential $\left(0.5 \mathrm{e}^{-1}\right)$, red: negative potential $\left(-0.04 \mathrm{e}^{-1}\right)$.

EF $\left(\mathrm{VA} \AA^{-1}\right)$

the DOS and decreases the HLG. The large decrease of band gap from 1.486 to $0.218 \mathrm{eV}$, at the high field, is facilitated to have high electrical conductivity. Also, the EF polarizes the molecule; in consequence the dipole moment of the molecule increases from 1.31 to $35.9 \mathrm{D}$. The $I-V$ characteristic curve is found very symmetric for both directions of applied EFs; it explicitly shows the nonlinear behavior of TTF based molecule. Further, the significant $I-V$ characteristic details of the molecule give an idea to tune the molecule for appropriate biasing voltages for the operation of molecular devices. Over all, the terminal groups and the central redox group of TTF unit of the molecular wire are found to be very sensitive to applied EF compared with the molecular region. The structural confirmation, charge density distribution, and electrostatic properties of TTF obtained in the study may support design of several kinds of molecular wires based on TTF and its derivatives.

\section{Conflict of Interests}

The authors declare that there is no conflict of interests regarding the publication of this paper.

\section{References}

[1] M. Rieth and W. Schommers, Handbook of Theoretical and Computational Nanotechnology, vol. 10, American Scientific Publishers, 2006.

[2] J. M. Seminario, Theoretical and Computational Chemistry, Elsevier, 2007.

[3] W. Haiss, H. van Zalinge, D. Bethell, J. Ulstrup, D. J. Schiffrin, and R. J. Nichols, "Thermal gating of the single molecule conductance of alkanedithiols," Faraday Discussions, vol. 131, pp. 253-264, 2006. 
[4] F. Chen, J. Hihath, Z. Huang, X. Li, and N. J. Tao, "Measurement of single-molecule conductance," Annual Review of Physical Chemistry, vol. 58, no. 1, pp. 535-564, 2007.

[5] A. Irfan, A. G. Al-Sehemi, and A. M. Asiri, "Theoretical investigations of the charge transfer properties in oligothiophene derivatives," Journal of Theoretical and Computational Chemistry, vol. 11, no. 3, article 631, 2012.

[6] L. Luo, S. H. Choi, and C. D. Frisbie, "Probing hopping conduction in conjugated molecular wires connected to metal electrodes," Chemistry of Materials, vol. 23, no. 3, pp. 631-645, 2011.

[7] X. Zeng, C. Wang, M. R. Bryce et al., "Functionalized $8 \mathrm{~nm}$ long aryleneethynylene molecular wire with alkyne termini," European Journal of Organic Chemistry, no. 31, pp. 5244-5249, 2007.

[8] A. Aviram and M. A. Ratner, "Molecular rectifiers," Chemical Physics Letters, vol. 29, no. 2, pp. 277-283, 1974.

[9] A. Aviram, C. Joachim, and M. Pomerantz, "Evidence of switching and rectification by a single molecule effected with a scanning tunneling microscope," Chemical Physics Letters, vol. 146, pp. 490-495, 1988.

[10] J. M. Tour, W. A. Reinerth, L. Jones II et al., "Recent advances in molecular scale electronics," Annals of the New York Academy of Sciences, vol. 852, pp. 197-204, 1998.

[11] Y. W. Li, J. H. Yao, Z. G. Zou, J. W. Yang, and S. R. Le, “Theoretical study of the electron transport through aromatic molecular wires with different levels of conjugation," Computational and Theoretical Chemistry, vol. 976, pp. 135-140, 2011.

[12] S. Creager, C. J. Yu, C. Bamdad et al., "Electron transfer at electrodes through conjugated "molecular wire" bridges," Journal of the American Chemical Society, vol. 121, no. 5, pp. 1059-1064, 1999.

[13] J. Ferraris, D. O. Cowan, V. Walatka Jr., and J. H. Perlstein, "Electron transfer in a new highly conducting donor-acceptor complex," Journal of the American Chemical Society, vol. 95, no. 3, pp. 948-949, 1973.

[14] A. Amadei, M. D’Abramo, A. D. Nola, A. Arcadi, G. Cerichelli, and M. Aschi, "Theoretical study of intramolecular charge transfer in $\pi$-conjugated oligomers," Chemical Physics Letters, vol. 434, no. 4-6, pp. 194-199, 2007.

[15] F. Wudl and M. L. Kaplan, "2,2' Bi-1,3-dithiolylidene (tetrathiafulvalene, TTF) and its radical cation derivatives," Inorganic Syntheses, vol. 19, pp. 27-30, 1979.

[16] F. Wudl, D. Wobschall, and E. J. Hufnagel, "Electrical conductivity by the bis-1,3-dithiole-bis-1,3-dithiolium system," Journal of the American Chemical Society, vol. 94, no. 2, pp. 670-672, 1972.

[17] M. Bendikov, F. Wudl, and D. F. Perepichka, "Tetrathiafulvalenes, oligoacenenes, and their buckminsterfullerene derivatives: the brick and mortar of organic electronics," Chemical Reviews, vol. 104, no. 11, pp. 4891-4945, 2004.

[18] M. Iyoda, M. Hasegawa, and Y. Miyake, "Bi-TTF, bis-TTF, and related TTF oligomers," Chemical Reviews, vol. 104, no. 11, pp. 5085-5113, 2004.

[19] P. Frère and P. J. Skabara, "Salts of extended tetrathiafulvalene analogues: relationships between molecular structure, electrochemical properties and solid state organisation," Chemical Society Reviews, vol. 34, no. 1, pp. 69-98, 2005.

[20] X. Li, J. He, J. Hihath, B. Xu, S. M. Lindsay, and N. Tao, "Conductance of single alkanedithiols: conduction mechanism and effect of molecule-electrode contacts," Journal of the American Chemical Society, vol. 128, no. 6, pp. 2135-2141, 2006.
[21] W. Sheng, Z. Y. Li, Z. Y. Ning, Z. H. Zhang, Z. Q. Yang, and H. Guo, "Quantum transport in alkane molecular wires: effects of binding modes and anchoring groups," The Journal of Chemical Physics, vol. 131, no. 24, Article ID 244712, 2009.

[22] A. Gorgues, P. Hudhomme, and M. Salle, "Highly functionalized tetrathiafulvalenes: riding along the synthetic trail from electrophilic alkynes," Chemical Reviews, vol. 104, no. 11, pp. 5151-5184, 2004.

[23] R. F. W. Bader, Atoms in Molecules-A Quantum Theory, Clarendon Press, Oxford, UK, 1990.

[24] E. S. Kryachko and E. Ludena, Density Functional Theory of Atoms of Many Electron System, Kluwer Academic, New York, NY, USA, 1990.

[25] J. M. Seminario, Recent Development and Applications of Modern Density Functional Theory, Elesvier, New York, NY, USA, 1996.

[26] D. Rai, A. D. Kulkarni, S. P. Gejji, and R. K. Pathak, "Methanol clusters $\left(\mathrm{CH}_{3} \mathrm{OH}\right)_{n}, \mathrm{n}=3-6$ in external electric fields: density functional theory approach," Journal of Chemical Physics, vol. 135, no. 2, Article ID 024307, 2011.

[27] M. Das, "Electron transfer through non-hydrogen and hydrogen bonded intermolecular tunnel junctions: a computational study," Journal of Theoretical and Computational Chemistry, vol. 11, p. 997, 2012.

[28] M. J. Frisch, G. W. Trucks, H. B. Schlegel et al. Gaussian Inc., Pittsburgh, Pa, USA, 2003.

[29] A. D. Becke, "Density-functional thermochemistry. III. The role of exact exchange," The Journal of Chemical Physics, vol. 98, no. 7, pp. 5648-5652, 1993.

[30] P. J. Hay and W. R. Wadt, "Ab initio effective core potentials for molecular calculations. Potentials for the transition metal atoms Sc to Hg," The Journal of Chemical Physics, vol. 82, no. 1, pp. 270283, 1985.

[31] Y. Yang, M. N. Weave, and K. M. Merz Jr., "Assessment of the "6-31+G** + LANL2DZ" mixed basis set coupled with density functional theory methods and the effective core potential: prediction of heats of formation and ionization potentials for first-row-transition-metal complexes," The Journal of Physical Chemistry A, vol. 113, no. 36, pp. 9843-9851, 2009.

[32] H. B. Schlegel, "Optimization of equilibrium geometries and transition structures," Journal of Computational Chemistry, vol. 3, no. 2, pp. 214-218, 1982.

[33] F. W. Biegler-könig, R. F. W. Bader, and T. H. Tang, "Calculation of the average properties of atoms in molecules. II," Journal of Computational Chemistry, vol. 3, no. 3, pp. 317-328, 1982.

[34] A. Frish, E. Lecn, A. B. Nielson, A. J. Holder, R. D. Roy Dennigton, and T. A. Keith Gaussian, $<$ ?tex cmt="Please provide more information for this reference."?> Inc., Pittsburgh, Pa, USA, 2003.

[35] N. O. Boyle, GaussSum, Revision 2.1, http://GaussSum.sf.net.

[36] J. M. Seminario, A. G. Zacarias, and J. M. Tour, "Molecular alligator clips for single molecule electronics. Studies of group 16 and isonitriles interfaced with Au contacts," Journal of the American Chemical Society, vol. 121, no. 2, pp. 411-416, 1999.

[37] Y. Zhang, Y. Ye, Y. Li, X. Yin, H. Liu, and J. Zhao, "Ab initio investigations of quaterthiophene molecular wire under the interaction of external electric field," Journal of Molecular Structure: THEOCHEM, vol. 802, pp. 53-58, 2007.

[38] Y. Ye, M. Zhang, H. Liu, X. Liu, and J. Zhao, "Theoretical investigation on the oligothienoacenes under the influence of external electric field," Journal of Physics and Chemistry of Solids, vol. 69, no. 11, pp. 2615-2621, 2008. 
[39] A. Johanasson and S. Stafstrom, "Interactions between molecular wires and a gold surface," Chemical Physics Letters, vol. 322, no. 5, pp. 301-306, 2000.

[40] P. Srinivasan, A. D. Stephen, and P. Kumaradhas, "Effect of gold atom contact in conjugated system of one dimensional octane dithiolate based molecular wire: a theoretical charge density study," Journal of Molecular Structure: THEOCHEM, vol. 910, no. 1-3, pp. 112-121, 2009.

[41] J. Li, T. Zhu, C. J. Cramer, and D. G. Truhlar, "New class IV charge model for extracting accurate partial charges from wave functions," Journal of Physical Chemistry A, vol. 102, no. 10, pp. 1820-1831, 1998.

[42] D. Cremer and E. Kraka, "A description of the chemical bond in terms of local properties of electron density and energy, in conceptual approaches in quantum chemistry-models and applications," Croatia Chemica Acta, vol. 57, pp. 1259-1281, 1984.

[43] D. J. R. Duarte, G. L. Sosa, and N. M. Peruchena, "Nature of halogen bonding. A study based on the topological analysis of the Laplacian of the electron charge density and an energy decomposition analysis," Journal of Molecular Modeling, vol. 19, no. 5, pp. 2035-2041, 2013.

[44] G. V. Gibbs, O. Tamada, M. B. Boisen Jr., and F. C. Hill, "Laplacian and bond critical point properties of the electron density distributions of sulfide bonds: a comparison with oxide bonds," American Mineralogist, vol. 84, no. 3, pp. 435-446, 1999.

[45] D. Rai, A. D. Kulkarni, S. P. Gejji, and R. K. Pathak, "Is high electric field capable of selectively inducing a covalent-like bond between polar and non-polar molecular species?" Theoretical Chemistry Accounts, vol. 123, pp. 501-511, 2009.

[46] R. G. A. Bone and R. F. W. Bader, "Identifying and analyzing intermolecular bonding interactions in van der Waals molecules," Journal of Physical Chemistry, vol. 100, no. 26, pp. 10892-10911, 1996.

[47] V. R. Hathwar, A. K. Paul, S. Natarajan, and T. N. Guru Row, "Charge density analysis of a pentaborate ion in an ammonium borate: toward the understanding of topological features in borate minerals," Journal of Physical Chemistry A, vol. 115, no. 45, pp. 12818-12825, 2011.

[48] R. F. W. Bader and H. Essen, "The characterization of atomic interactions," The Journal of Chemical Physics, vol. 80, p. 1943, 1984.

[49] G. V. Gibbs, R. T. Downs, D. F. Cox et al., "Experimental bond critical point and local energy density properties determined for $\mathrm{Mn}-\mathrm{O}, \mathrm{Fe}-\mathrm{O}$, and $\mathrm{Co}-\mathrm{O}$ bonded interactions for tephroite, $\mathrm{Mn}_{2} \mathrm{SiO}_{4}$, fayalite, $\mathrm{Fe}_{2} \mathrm{SiO}_{4}$, and $\mathrm{Co}_{2} \mathrm{SiO}_{4}$ olivine and selected organic metal complexes: comparison with properties calculated for non-transition and transition metal M-O bonded interactions for silicates and oxides," The Journal of Physical Chemistry A, vol. 112, no. 37, pp. 8811-8823, 2008.

[50] H. Jacobsen, "Kinetic energy density and covalent bonding-a complementary analysis at the border of bond and no bond," Dalton Transactions, vol. 39, no. 23, pp. 5426-5428, 2010.

[51] R. S. Mulliken, "Electronic population analysis on LCAO-MO molecular wave functions. I," The Journal of Chemical Physics, vol. 23, no. 10, pp. 1833-1840, 1955.

[52] C. Campañá, B. Mussard, and T. K. Woo, "Electrostatic potential derived atomic charges for periodic systems using a modified error functional," Journal of Chemical Theory and Computation, vol. 5, no. 10, pp. 2866-2878, 2009.

[53] F. Martin and H. Zipse, "Charge distribution in the water molecule - a comparison of methods," Journal of Computational Chemistry, vol. 26, no. 1, pp. 97-105, 2005.
[54] P. A. Singh and J. Kollman, "An approach to computing electrostatic charges for molecules," Journal of Computational Chemistry, vol. 5, pp. 129-145, 1984.

[55] G. S. Maciel and E. Garcia, "Charges derived from electrostatic potentials: exploring dependence on theory and geometry optimization levels for dipole moments," Chemical Physics Letters, vol. 409, pp. 29-33, 2005.

[56] J. M. Seminario and L. Yan, "Ab initio analysis of electron currents in thioalkanes," International Journal of Quantum Chemistry, vol. 102, no. 5, pp. 711-723, 2005.

[57] P. Wang, C. N. Moorefield, S. Li, S.-H. Hwang, C. D. Shreiner, and G. R. Newkome, "TerpyridineCuII-mediated reversible nanocomposites of single-wall carbon nanotubes: towards metallo-nanoscale architectures," Chemical Communications, no. 10, pp. 1091-1093, 2006.

[58] J. M. Seminario, A. G. Zacarias, and P. A. Derosa, "Theoretical analysis of complementary molecular memory devices," The Journal of Physical Chemistry A, vol. 105, no. 5, pp. 791-795, 2001.

[59] J. M. Seminario, R. A. Araujo, and L. Yan, "Negative differential resistance in metallic and semiconducting clusters," Journal of Physical Chemistry B, vol. 108, no. 22, pp. 6915-6918, 2004.

[60] J. M. Seminario, C. de la Cruz, P. A. Derosa, and L. Yan, "Nanometer-size conducting and insulating molecular devices," The Journal of Physical Chemistry B, vol. 108, no. 46, pp. 1787917885, 2004.

[61] L. Yan and J. M. Seminario, "Electronic structure and electron transport characteristics of a cobalt complex," The Journal of Physical Chemistry A, vol. 109, no. 30, pp. 6628-6633, 2005.

[62] Y. Li, J. Zhao, X. Yin, H. Liu, and G. Yin, "Conformational analysis of diphenylacetylene under the influence of an external electric field," Physical Chemistry Chemical Physics, vol. 9, no. 10, pp. 1186-1193, 2007.

[63] C. Xia, Y. Zhang, and D. Liu, "Effect of torsion angle on the rectifying performance in the donor-bridge-acceptor single molecular device," Journal of Theoretical and Computational Chemistry, vol. 11, p. 735, 2012.

[64] W. B. Davis, W. A. Svec, M. A. Ratner, and M. R. Wasielewski, "Molecular-wire behaviour in p-phenylenevinylene oligomers," Nature, vol. 396, no. 6706, pp. 60-63, 1998.

[65] Y. Zhou, K. Tan, and X. Lu, "Insights into the solvato-/thermopromoted intramolecular electron transfer in a TTF- $\sigma$-TCNQ dyad with an extremely low homo-lumo gap," Journal of Theoretical and Computational Chemistry, vol. 11, no. 3, p. 599, 2012.

[66] R. J. Magyar, S. Tretiak, Y. Gao, H.-L. Wang, and A. P. Shreve, "A joint theoretical and experimental study of phenylene-acetylene molecular wires," Chemical Physics Letters, vol. 401, no. 1-3, pp. 149-156, 2005.

[67] S. Hong, R. Reifenberger, W. Tian, S. Datta, J. I. Henderson, and C. P. Kubiak, "Molecular conductance spectroscopy of conjugated, phenyl-based molecules on $\mathrm{Au}(111)$ : the effect of end groups on molecular conduction," Superlattices and Microstructures, vol. 28, no. 4, pp. 289-303, 2000.

[68] D. Farmanzadeh and Z. Ashtiani, "Theoretical study of a conjugated aromatic molecular wire," Structural Chemistry, vol. 21, no. 4, pp. 691-699, 2010.

[69] R. K. Pathak and S. R. Gadre, "Maximal and minimal characteristics of molecular electrostatic potentials," The Journal of Chemical Physics, vol. 93, no. 3, pp. 1770-1773, 1990.

[70] P. Politzer and D. G. Thruhlar, Chemical Applications of Atomic and Molecular Electrostatic Potential, Plenum Press, New York, NY, USA, 1981. 
[71] B. Hu, C. Yao, and Q. Wang, "Electron-withdrawing substituted btd-based derivative: electronic and optical properties, charge transfer, stability study," Journal of Theoretical and Computational Chemistry, vol. 10, no. 6, pp. 829-838, 2011.

[72] J. Tomasi, B. Mennucci, M. Cammi, S. Murray, and K. D. Sen, Eds., Molecular Electrostatic Potentials. Concepts and Application, Elsevier, Amsterdam, The Netherlands, 1996.

[73] S. R. Gadre, Computational Chemistry: Reviews of Current Trends, World Scientific, Singapore, 2000.

[74] Y. Ye, M. Zhang, and J. Zhao, "Ab initio investigations on three isomers of polyacetylene under the interaction of the external electric field," Journal of Molecular Structure: THEOCHEM, vol. 822, no. 1-3, pp. 12-20, 2007.

[75] D. M. Bishop, "Molecular vibrational and rotational motion in static and dynamic electric fields," Reviews of Modern Physics, vol. 62, no. 2, pp. 343-374, 1990.

[76] B. Kirtman, B. Champagne, and D. M. J. Bishop, "Electric field simulation of substituents in donor-acceptor polyenes: a comparison with ab initio predictions for dipole moments, polarizabilities, and hyperpolarizabilities," Journal of the American Chemical Society, vol. 122, no. 33, pp. 8007-8012, 2002.

[77] N. C. Greenham, R. H. Friend, H. Enhrenreich, and F. Spacepen, Solid State Physics, Academic Press, San Diego, Calif, USA, 1995.

[78] J. M. Seminario, A. G. Zacarias, and J. M. Tour, "Molecular current-voltage characteristics," The Journal of Physical Chemistry A, vol. 103, no. 39, pp. 7883-7887, 1999.

[79] R. Landauer, "Can a length of perfect conductor have a resistance?” Physics Letters A, vol. 85, no. 2, pp. 91-93, 1981.

[80] J. G. Kushmerick, J. Naciri, J. C. Yang, and R. Shashidhar, "Conductance scaling of molecular wires in parallel," Nano Letters, vol. 3, no. 7, pp. 897-900, 2003.

[81] D. Farmanzadeh and Z. Ashtiani, "Theoretical study of a conjugated aromatic molecular wire," Structural Chemistry, vol. 21, no. 4, pp. 691-699, 2010.

[82] M. D. Ganji and A. Mir-Hashemi, "Ab initio investigation of the I-V characteristics of the butadiene nano-molecular wires: a light-driven molecular switch," Physics Letters, Section A: General, Atomic and Solid State Physics, vol. 372, no. 17, pp. 3058-3063, 2008.

[83] K. Selvaraju, M. Jothi, and P. Kumaradhas, "A charge density analysis on quarter thiophene molecular nanowire under applied electric field: a theoretical study," Journal of Computational and Theoretical Nanoscience, vol. 10, no. 2, pp. 357-367, 2013. 



Submit your manuscripts at http://www.hindawi.com


\section{The Scientific World Journal}
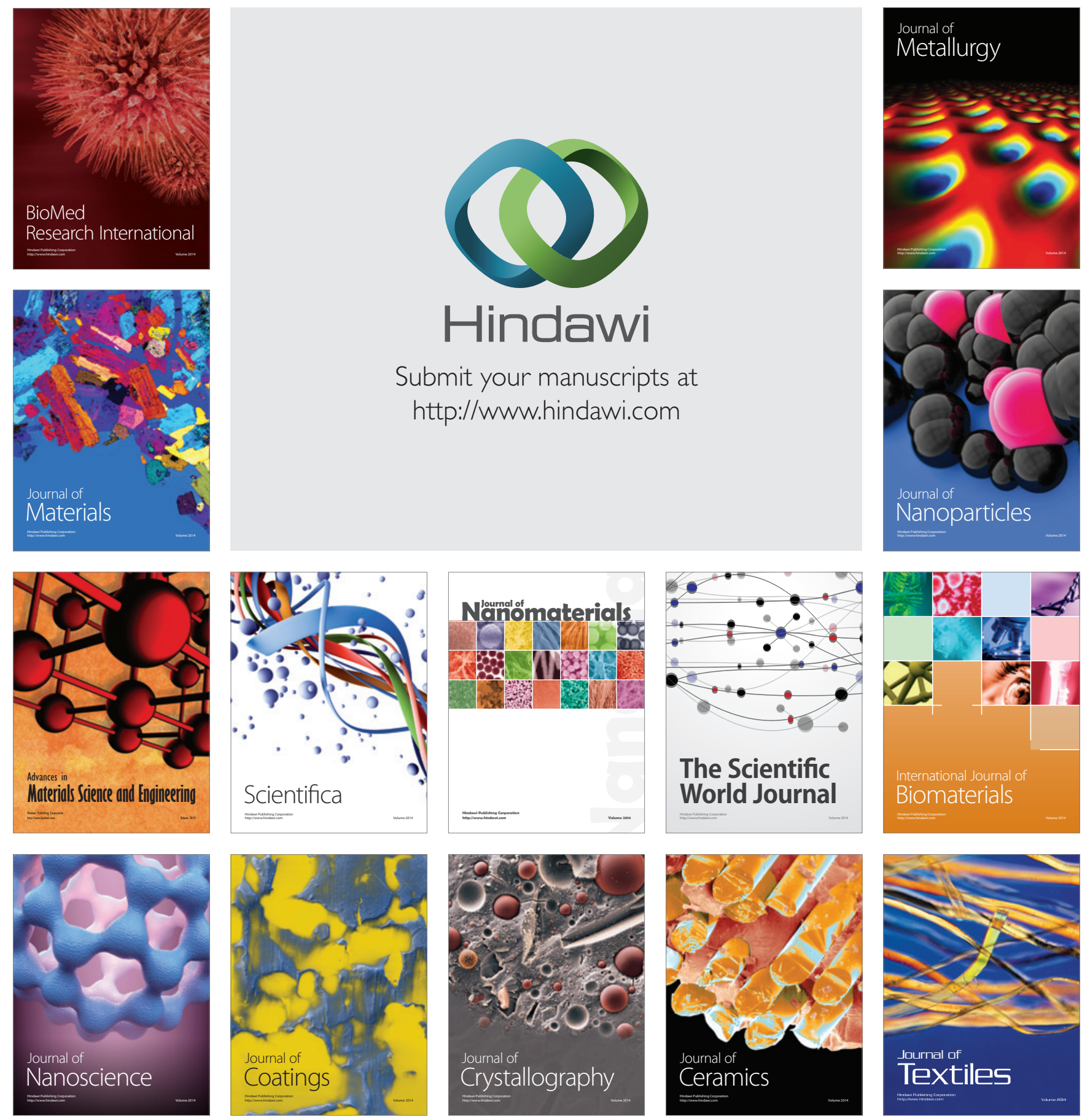